\title{
Synthetic Control Of Thorium Polyoxo-Clusters In Metal-Organic Frameworks Toward New Thorium-Based Materials
}

Peng $\mathrm{Li}^{\dagger}$, Xingjie Wang $^{\S, \dagger}$, Ken-ichi Otake $e^{\dagger}$, Jiafei Lyu ${ }^{\perp, \dagger}$, Sylvia L. Hanna, ${ }^{\dagger}$ Timur Islamoglu, ${ }^{\dagger}$ Omar K. Farha ${ }^{\dagger, *}$

$\dagger$ Department of Chemistry and International Institute of Nanotechnology, Northwestern University, 2145 Sheridan Road, Evanston, Illinois 60208, United States

$\S$ School of Chemistry and Chemical Engineering, South China University of Technology, Guangzhou 510640, PR China

${ }^{\perp}$ Department of Pharmaceutical Engineering, School of Chemical Engineering and Technology, Tianjin University, Tianjin 300350, China.

\section{Corresponding Author}

*(OKF) Email: $\underline{\text { o-farha@northwestern.edu }}$ 


\section{Table of Contents}

Table S1. Crystal data and structure refinement for Th-NU-1008 and Th-NU-1011 . $\mathrm{S} 3$

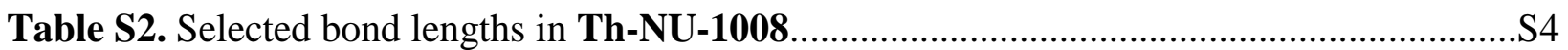

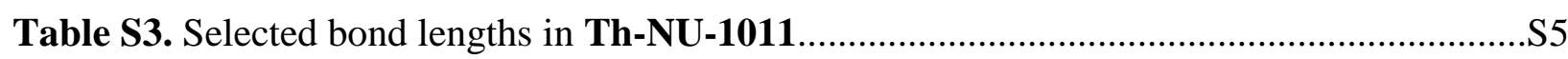

Figure S1. Optical images of single crystals of Th-NU-1008 and Th-NU-1011_......................S6

Figure S2. TGA curves of Th-NU-1008 and Th-NU-1011 ..................................................S6

Figure S3. PXRD patterns of Th-NU-1008 (top) and Th-NU-1011 (bottom) in air, water, acid,

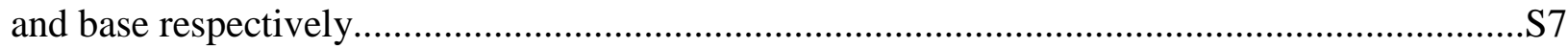

Figure S4. PXRD patterns of unknown phase, pure phase and mixture phase of Th-NU-1008

and Th-NU-1011, and phase transformation from Th-NU-1011 to Th-NU-1008......................S 8 
Table S1. Crystal data and structure refinement for Th-NU-1008 and Th-NU-1011

\begin{tabular}{|c|c|c|}
\hline MOF & Th-NU-1008 & Th-NU-1011 \\
\hline CCDC-No. & 1875458 & 1882912 \\
\hline Empirical formula & $\mathrm{C}_{71.6} \mathrm{H}_{32} \mathrm{Br}_{4} \mathrm{O}_{30} \mathrm{Th}_{6}$ & $\mathrm{C}_{71} \mathrm{H}_{38} \mathrm{Br}_{4} \mathrm{NO}_{28.5} \mathrm{Th}_{4}$ \\
\hline Formula weight & 3084.05 & 2608.82 \\
\hline Temperature/K & $100(2)$ & $200(2)$ \\
\hline Crystal system & hexagonal & hexagonal \\
\hline Space group & $P 6 / \mathrm{mmm}$ & $P 6 / m c c$ \\
\hline $\mathrm{a} / \mathrm{A}$ & $41.2089(17)$ & $27.0704(8)$ \\
\hline $\mathrm{b} / \AA ̊$ & $41.2089(17)$ & $27.0704(8)$ \\
\hline$c / \AA$ & $12.2663(7)$ & $33.5896(12)$ \\
\hline$\alpha /^{\circ}$ & 90 & 90 \\
\hline$\beta /{ }^{\circ}$ & 90 & 90 \\
\hline$\gamma /{ }^{\circ}$ & 120 & 120 \\
\hline Volume $/ \AA^{3}$ & $18039.6(18)$ & $21316.9(15)$ \\
\hline $\mathrm{Z}$ & 3 & 6 \\
\hline$\rho_{\text {calc }} \mathrm{g} / \mathrm{cm}^{3}$ & 0.852 & 1.219 \\
\hline$\mu / \mathrm{mm}^{-1}$ & 12.818 & 15.043 \\
\hline $\mathrm{F}(000)$ & 4145.0 & 7194.0 \\
\hline Crystal size $/ \mathrm{mm}^{3}$ & $0.080 \times 0.040 \times 0.040$ & $0.080 \times 0.040 \times 0.040$ \\
\hline Radiation & $\mathrm{CuK} \alpha(\lambda=1.54178)$ & $\mathrm{CuK} \alpha(\lambda=1.54178)$ \\
\hline $2 \Theta$ range for data collection $/{ }^{\circ}$ & 4.952 to 120.252 & 3.768 to 122.88 \\
\hline Index ranges & $-43 \leq \mathrm{h} \leq 42,-45 \leq \mathrm{k} \leq 46,-8 \leq 1 \leq 13$ & $-30 \leq \mathrm{h} \leq 29,-29 \leq \mathrm{k} \leq 30,-38 \leq 1 \leq 32$ \\
\hline Reflections collected & 44486 & 77479 \\
\hline Independent reflections & $5075[$ Rint $=0.1027$, Rsigma $=0.0569]$ & $5631\left[R_{\text {int }}=0.0944, R_{\text {sigma }}=0.0375\right]$ \\
\hline Data/restraints/parameters & $5075 / 0 / 153$ & $5631 / 16 / 258$ \\
\hline Goodness-of-fit on $\mathrm{F}^{2}$ & 1.061 & 1.175 \\
\hline Final $\mathrm{R}$ indexes $[\mathrm{I}>=2 \sigma(\mathrm{I})]$ & $\mathrm{R} 1=0.0294, \mathrm{wR} 2=0.0674$ & $\mathrm{R}_{1}=0.0488, \mathrm{wR}_{2}=0.1543$ \\
\hline Final $\mathrm{R}$ indexes [all data] & $\mathrm{R} 1=0.0396, \mathrm{wR} 2=0.0698$ & $\mathrm{R}_{1}=0.0614, \mathrm{wR}_{2}=0.1654$ \\
\hline Largest diff. peak/hole / e $\AA^{-3}$ & $1.05 /-2.14$ & $1.33 /-1.43$ \\
\hline
\end{tabular}


Table S2. Selected bond lengths in Th-NU-1008

\begin{tabular}{|c|c|c|c|c|c|}
\hline Atom & Atom & Length/Å & Atom & Atom & Length/Å \\
\hline $\mathrm{O}(1)$ & $\operatorname{Th}(1)$ & $2.397(5)$ & $\operatorname{Th}(1)$ & $\operatorname{Th}(2)^{5}$ & $3.9784(3)$ \\
\hline $\mathrm{O}(2)$ & $\operatorname{Th}(2)$ & $2.470(6)$ & $\operatorname{Th}(1)$ & $\operatorname{Th}(2)^{3}$ & $3.9784(3)$ \\
\hline $\mathrm{O}(3)$ & $\operatorname{Th}(2)$ & $2.697(10)$ & $\operatorname{Th}(2)$ & $\operatorname{Th}(2)^{3}$ & $3.9145(6)$ \\
\hline $\mathrm{O}(4)$ & $\operatorname{Th}(2)$ & $2.466(5)$ & $\mathrm{O}(1)$ & $\operatorname{Th}(2)^{3}$ & $2.376(3)$ \\
\hline $\mathrm{O}(5)$ & $\operatorname{Th}(2)^{5}$ & $2.423(3)$ & $\mathrm{O}(1)$ & $\operatorname{Th}(2)$ & $2.376(3)$ \\
\hline $\mathrm{O}(5)$ & $\operatorname{Th}(2)^{3}$ & $2.423(3)$ & $\mathrm{O}(6)$ & $\operatorname{Th}(1)$ & $2.631(9)$ \\
\hline $\mathrm{O}(5)$ & $\operatorname{Th}(1)$ & $2.423(5)$ & $\mathrm{O}(7)$ & $\operatorname{Th}(1)$ & $2.461(4)$ \\
\hline $\operatorname{Th}(1)$ & $\operatorname{Th}(2)^{4}$ & $3.9784(3)$ & $\mathrm{O}(8)$ & $\operatorname{Th}(2)$ & $2.437(4)$ \\
\hline
\end{tabular}


Table S3. Selected bond lengths in Th-NU-1011

\begin{tabular}{|c|c|c|c|c|c|}
\hline Atom & Atom & Length/Å & Atom & Atom & Length/Å \\
\hline$\overline{\mathrm{Th}}(1)$ & $\mathrm{O}(1)$ & $2.260(7)$ & $\mathrm{Th}(1)$ & $\mathrm{O}(10)^{2}$ & $2.740(16)$ \\
\hline $\operatorname{Th}(1)$ & $\mathrm{O}(1)^{1}$ & $2.329(6)$ & $\operatorname{Th}(2)$ & $\mathrm{O}(1)$ & $2.273(7)$ \\
\hline $\operatorname{Th}(1)$ & $\mathrm{O}(9)^{2}$ & $2.333(15)$ & $\operatorname{Th}(2)$ & $\mathrm{O}(5)^{2}$ & $2.345(7)$ \\
\hline $\operatorname{Th}(1)$ & $\mathrm{O}(9)$ & $2.333(15)$ & $\operatorname{Th}(2)$ & $\mathrm{O}(5)$ & $2.345(7)$ \\
\hline $\operatorname{Th}(1)$ & $\mathrm{O}(6)^{2}$ & $2.381(7)$ & $\operatorname{Th}(2)$ & $\mathrm{O}(8)$ & $2.416(11)$ \\
\hline $\operatorname{Th}(1)$ & $\mathrm{O}(6)$ & $2.381(7)$ & $\operatorname{Th}(2)$ & $\mathrm{O}(8)^{2}$ & $2.416(11)$ \\
\hline $\operatorname{Th}(1)$ & $\mathrm{O}(7)$ & $2.408(16)$ & $\operatorname{Th}(2)$ & $\mathrm{O}(11)^{1}$ & $2.48(3)$ \\
\hline $\operatorname{Th}(1)$ & $\mathrm{O}(7)^{2}$ & $2.408(16)$ & $\operatorname{Th}(2)$ & $\mathrm{O}(3)^{2}$ & $2.540(16)$ \\
\hline $\operatorname{Th}(1)$ & $\mathrm{O}(11)$ & $2.45(3)$ & $\operatorname{Th}(2)$ & $\mathrm{O}(3)$ & $2.540(16)$ \\
\hline $\operatorname{Th}(1)$ & $\mathrm{O}(2)$ & $2.64(2)$ & $\operatorname{Th}(2)$ & $\mathrm{O}(4)$ & $2.64(2)$ \\
\hline $\operatorname{Th}(1)$ & $\mathrm{O}(10)$ & $2.740(16)$ & & & \\
\hline
\end{tabular}

\footnotetext{
${ }^{1} 1-X, 1-Y, 1-Z ;{ }^{2}+X,+Y, 1-Z ;{ }^{3} 1+Y-X,+Y, 1 / 2-Z ;$

${ }^{4} 1-Y+X,+X, 1-Z ;{ }^{5} 1+Y-X, 1-X, 1-Z ;{ }^{6} 1-X, 1-Y,+Z$
} 

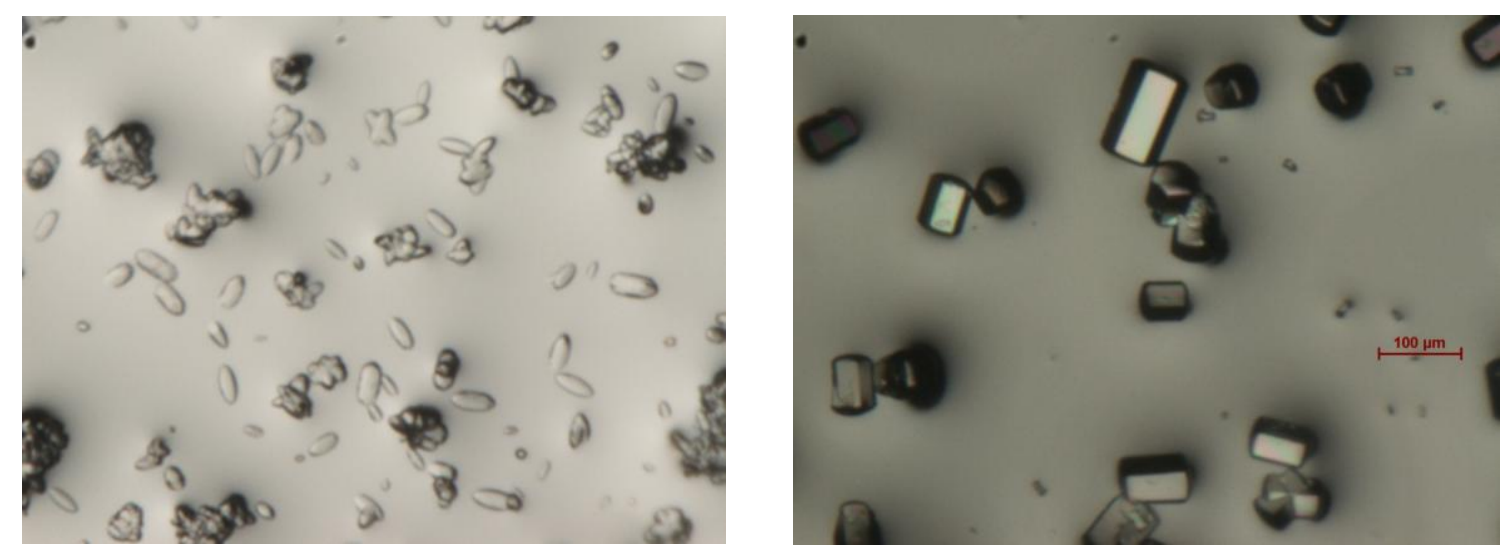

Figure S1. Optical images of single crystals of Th-NU-1008 (left) and Th-NU-1011 (right).

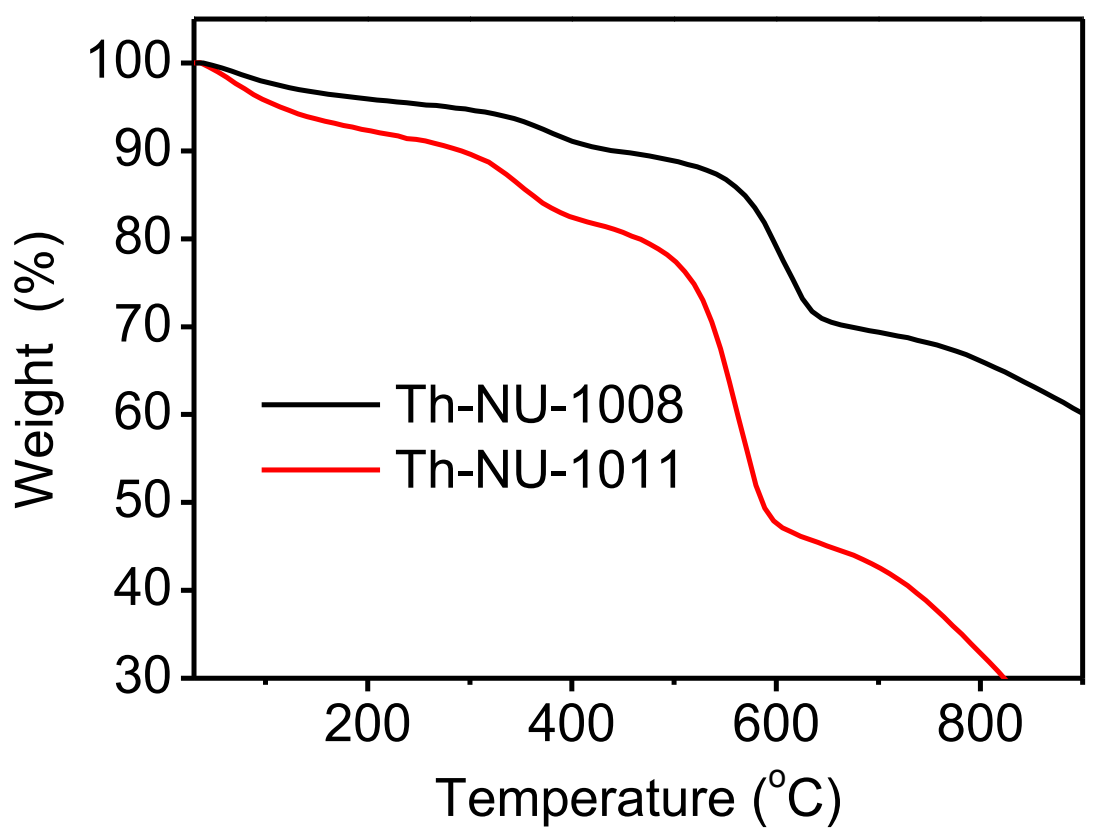

Figure S2. TGA curves of Sc-CO $\mathrm{CO}_{2}$ activated Th-NU-1008 (black) and Th-NU-1011 (red). 

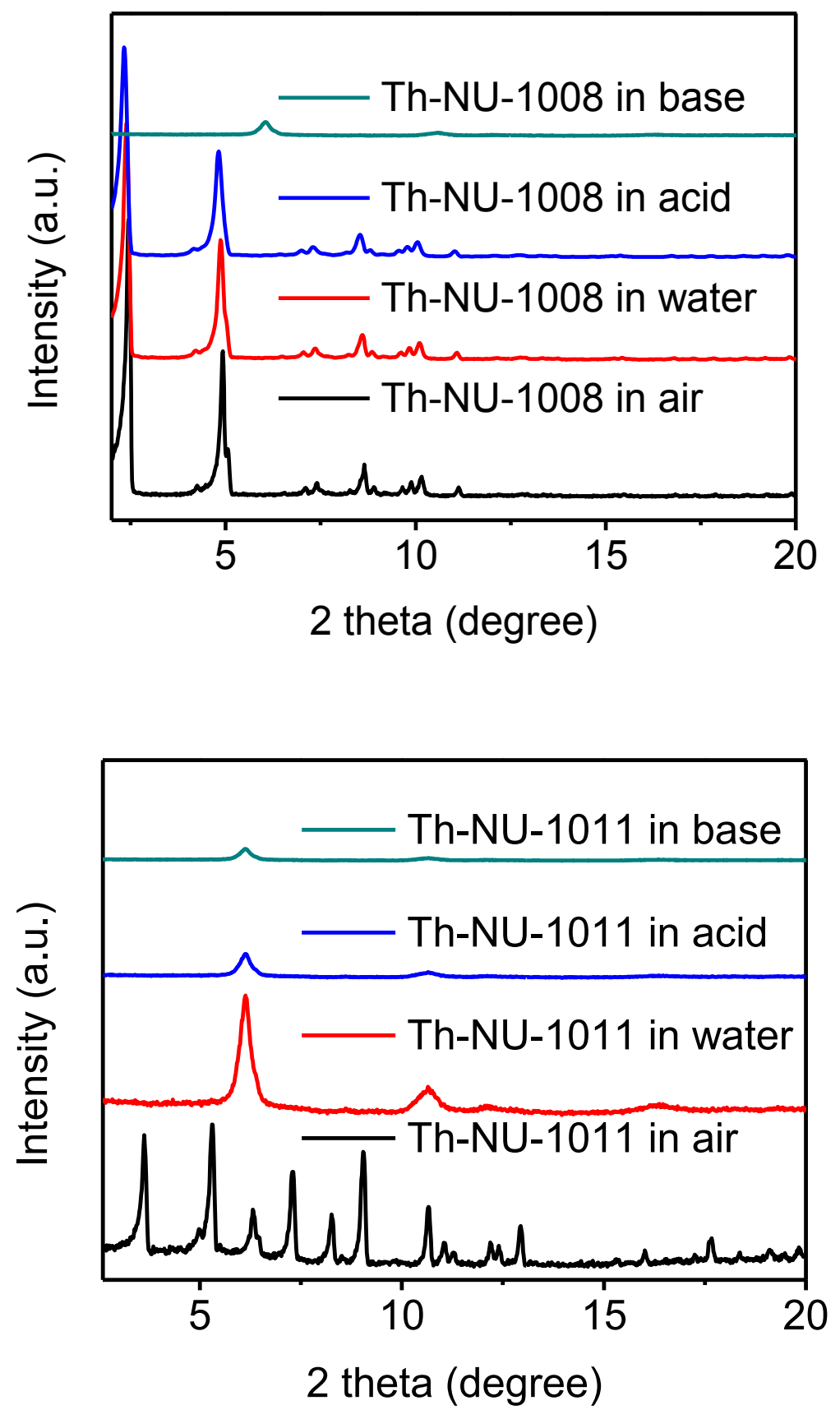

Figure S3. PXRD patterns of Th-NU-1008 (top) and Th-NU-1011 (bottom) in air, water, acid, and base respectively. 


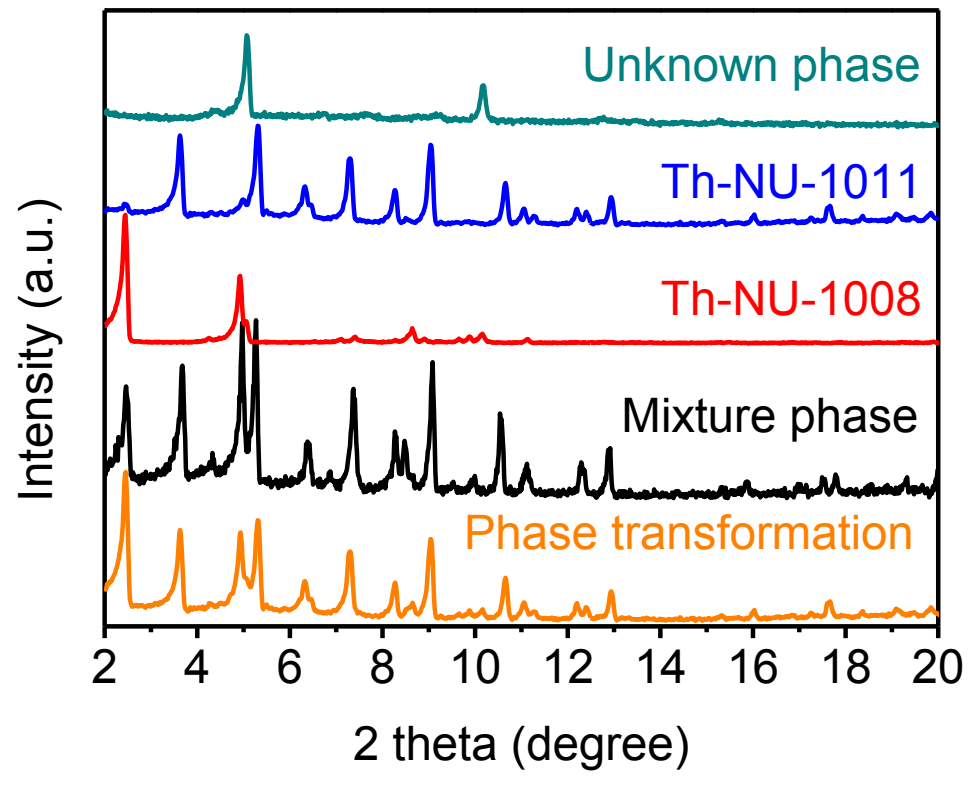

Figure S4. PXRD patterns of unknown phase, pure phase and mixture phase of Th-NU-1008 and Th-NU-1011, and phase transformation from Th-NU-1011 to Th-NU-1008. 